DRAFT VERSION JULY 30, 2021

Preprint typeset using $\mathrm{LT}_{\mathrm{E}} \mathrm{X}$ style emulateapj v. 03/07/07

\title{
TURBULENCE IN A THREE-DIMENSIONAL DEFLAGRATION MODEL FOR TYPE IA SUPERNOVAE: I. SCALING PROPERTIES
}

\author{
F. Ciaraldi-Schoolmann, W. Schmidt, J. C. Niemeyer, \\ Lehrstuhl für Astronomie und Astrophysik, Universität Würzburg, Am Hubland, D-97074 Würzburg, Germany
}

F. K. RÖPKE AND W. HiLlEBRANDT

Max-Planck-Institut für Astrophysik, Karl-Schwarzschild-Str. 1, D-85741 Garching, Germany

Draft version July 30, 2021

\begin{abstract}
We analyze the statistical properties of the turbulent velocity field in the deflagration model for Type Ia supernovae. In particular, we consider the question of whether turbulence is isotropic and consistent with the Kolmogorov theory at small length scales. Using numerical data from a high-resolution simulation of a thermonuclear supernova explosion (Röpke et al., 2007), spectra of the turbulence energy and velocity structure functions are computed. We show that the turbulent velocity field is isotropic at small length scales and follows a scaling law that is consistent with the Kolmogorov theory until most of the nuclear fuel is burned. At length scales greater than a certain characteristic scale that agrees with the prediction of Niemeyer and Woosley (1997), turbulence becomes anisotropic. Here, the radial velocity fluctuations follow the scaling law of the Rayleigh-Taylor instability, whereas the angular component still obeys Kolmogorov scaling. In the late phase of the explosion, this characteristic scale drops below the numerical resolution of the simulation. The analysis confirms that a subgrid-scale model for the unresolved turbulence energy is required for the consistent calculation of the flame speed in deflagration models of Type Ia supernovae, and that the assumption of isotropy on these scales is appropriate.
\end{abstract}

Subject headings: hydrodynamics — instabilities — methods: statistical — turbulence — Supernovae: general

\section{INTRODUCTION}

The mechanism of thermonuclear explosions of white dwarf (WD) stars, giving rise to Type Ia supernovae (SNe Ia) is still not understood in full detail. For three reasons, a key to this problem is the understanding of turbulent thermonuclear combustion in the deflagration phase - the phase of subsonic flame propagation which commences the explosion process. First, it is required for the correct modeling of the flame propagation in the deflagration model of SNe Ia (see Hillebrandt \& Niemeyer 2000, for a review of SN Ia explosion scenarios). Second, in alternative models, burning starts out in the deflagration mode, and this is an essential ingredient to the overall explosion process. Third, turbulence in the deflagration phase sets the conditions for a possible deflagration-todetonation transition (DDT) in the delayed detonation scenario (Röpke 2007; Woosley 2007). The necessary insight into the details of the turbulent combustion process, however, is hampered by the fact that full-star simulations of thermonuclear supernova explosions cannot resolve the structure of the deflagration flame. At the large scales accessible to simulations, the flame propagation is determined by flame instabilities and turbulence. These effects significantly boost the effective burning speed and, in the pure deflagration model of $\mathrm{SNe}$ Ia, lead to the flame acceleration required to explode the WD (Reinecke et al. 2002, Gamezo et al. 2003). In order to describe the flame propagation in such simulations, the interaction of the flame with turbulent velocity fluctuations has to be modeled. These modeling approaches yield an effective flame propagation speed on the numerically resolved scalesthe so-called turbulent burning speed.

The problem of calculating this turbulent flame propagation speed in three-dimensional simulations of thermonuclear su- pernova explosions ${ }^{1}$ has been the subject of a lively debate. One school of thought holds the view that the effective propagation speed in the flamelet regime would naturally be given by the velocity scale $v_{\mathrm{RT}}(\ell) \propto\left(g_{\text {eff }} \ell\right)^{1 / 2}$ associated with the Rayleigh-Taylor (RT) instability induced by buoyancy in the gravitational field $g_{\text {eff }}$ for any length scale $\ell$ (Sharp 1984). As a subgrid scale model $(\ell=\Delta$, where $\Delta$ is the numerical cutoff length), this scaling relation is easily implemented and appears to be motivated by the basic physics of thermonuclear combustion in Type Ia supernovae (Gamezo et al. 2003). In opposition to this view, Niemeyer \& Hillebrandt (1995) and Niemeyer \& Kerstein (1997) argued that inevitably turbulent velocity fluctuations $v^{\prime}(\ell)$ are dominated by the turbulent cascade at length scales $\ell$ small compared to the scale of energy injection by the RT instability and, hence, should follow the Kolmogorov scaling $v^{\prime}(\ell) \propto \ell^{1 / 3}$. Niemeyer \& Woosley (1997) estimated the transition length $\ell_{\mathrm{K} / \mathrm{RT}}$ between the RTdominated length scales (the "large scales" $\ell \gtrsim \ell_{\mathrm{K} / \mathrm{RT}}$ ) and the regime of the turbulent cascade (the "small scales" $\ell \lesssim \ell_{\mathrm{K} / \mathrm{RT}}$ ) to be of the order $10 \mathrm{~km}$. Since the cutoff length $\Delta$ in contemporary numerical simulation is comparable to or less than $\ell_{\mathrm{K} / \mathrm{RT}}$, it follows that a subgrid scale (SGS) model for the consistent calculation of the turbulent flame speed has to be based on the turbulence energy associated with the length scale $\Delta$, which is determined by the the dynamics of the turbulent cascade. Such an SGS model was proposed by Niemeyer \& Hillebrandt (1995) and further developed by Schmidt et al. (2006). It is not clear a priori that the turbulence to be captured by the SGS ansatz is of Kolmogorov-type and therefore the approach of Schmidt et al. (2006) is not based on this assumption. However, Kolmogorov scaling is an obvious possi-

\footnotetext{
${ }^{1}$ For a review, see Röpke \& Schmidt 2008.
} 
bility that has to be considered.

The best way to gain insight into the properties of turbulence in the deflagration stage is to analyze it directly in highresolution simulations of the deflagration model. Here the effects of gravity and spherical expansion of the background are naturally accounted for. In this paper, we present such an $a$ posteriori analysis of turbulence based on data from a recent numerical simulation (Röpke et al. 2007) which was carried out on a very large grid $\left(1024^{3}\right.$ cells $)$. The characteristics of this deflagration model as well as derived synthetic observables are in reasonable agreement with the observations of dimmer (but still normal) observed SNe Ia (Röpke et al. 2007). Therefore, our analysis is based on data from a model that is expected to give a realistic picture of turbulence in SNe Ia. The failure of the pure deflagration scenario to reproduce the brighter end of the SN Ia sample does not limit the significance of our results as in all alternative scenarios currently under discussion a similar deflagration phase initiates the explosion and sets the stage for the later evolution.

In the simulation analyzed here, the co-moving grid technique introduced by Röpke (2005) allowed for a very small initial cutoff length $\Delta \sim 1 \mathrm{~km}$ in the inner regions of the exploding white dwarf. Although $\Delta$ was gradually increased in the course of the simulation, it was possible to investigate the behaviour of turbulent velocity fluctuations at length scales $\ell \sim 10 \mathrm{~km}$ by means of computing kinetic energy spectrum functions of the velocity field in subdomains selected by an appropriate window function. The results indicated Kolmogorov scaling (Röpke et al. 2007). In this article, we refine this analysis by decomposing the velocity field into radial and angular components. In addition, we subtract the spherically averaged radial velocity in order to separate the velocity fluctuations from the mean radial expansion of the white dwarf. For both components of the fluctuating velocity field, a Fourier analysis is carried out to compute kinetic energy spectra and to investigate possible anisotropies. Since Fourier transforms are distorted by the non-periodic boundary of the computational domain, we calculate structure functions of the fluctuating velocity field to obtain reliable estimates of the scaling properties for the whole dynamical range of the simulation.

The methodology of our analysis is explained in detail in the following section. As will be shown in Section 3, RT scaling is found for the radial fluctuating velocity component at length scales greater than $\ell_{\mathrm{K} / \mathrm{RT}}$, whereas Kolmogorov scaling applies for smaller length scales. In close agreement with the prediction by Niemeyer \& Woosley (1997), the numerically determined value of $\ell_{\mathrm{K} / \mathrm{RT}}$ is about $15 \mathrm{~km}$ after the onset of the explosion. The angular velocity component, on the other hand, closely follows the Kolmogorov scaling law at all length scales. Furthermore, the velocity fluctuations are nearly isotropic at length scales smaller than $\ell_{\mathrm{K} / \mathrm{RT}}$. In the last section, we discuss possible caveats of our analysis and comment on the implications for numerical simulations of Type Ia supernovae.

\section{ANALYSIS OF THE TURBULENT VELOCITY FIELD}

For the statistical analysis of turbulence, we have to take into account that the velocity field is a superposition of turbulent velocity fluctuations and the bulk expansion of the white dwarf. To estimate the bulk expansion, we average the radial component of the velocity field over spherical shells of dis- crete radii $r_{i}$ :

$$
\bar{v}\left(r_{i}\right)=\frac{1}{N_{i}} \cdot \sum_{j=1}^{N_{i}} \mathbf{v}\left(\mathbf{r}_{i} j\right) \cdot \mathbf{e}_{r}\left(\mathbf{r}_{i} j\right),
$$

where $r_{i}^{2}=i \Delta^{2}(t)$ is an integer multiple of the squared size $\Delta(t)$ of the grid cells at time $t$. The sum is over all cells in the cubic grid that are located at the distance $r_{i}$ from the center, and $N_{i}$ is the corresponding number of cells. The unit vector in radial direction at the position $\mathbf{r}_{i} j$ of the $j$-th cell in the $i$ th shell is denoted by $\mathbf{e}_{r}\left(\mathbf{r}_{i} j\right)$. Using this estimate, we subtract the spherically averaged component $\bar{v}\left(r_{i}\right)$ from the original velocity field. In the following, it is understood that the symbol $\mathbf{v}$ refers to the fluctuating part of the velocity field.

Since the RT-Instability evolves in the direction of gravity, we perform all computations with velocity components parallel and perpendicular to the gravitational field. These components correspond to the radial and angular directions, because the gravitational field is assumed to be spherically symmetric in the simulation. Thus, we define $\mathbf{v}_{\|}:=v_{r} \mathbf{e}_{r}$, where $\mathbf{e}_{r}=\mathbf{r} / r$ and $v_{r}=\mathbf{v} \cdot \mathbf{e}_{r}$, and $\mathbf{v}_{\perp}:=\mathbf{v}-\mathbf{v}_{\|}$. The corresponding energy spectrum functions are obtained by integrating the kinetic energy per unit mass over spheres of radius $k$ in Fourier space:

$$
\begin{aligned}
E_{\|}(k) & =\frac{1}{2} \oint \mathrm{d} \Omega_{k} k^{2}\left|\hat{\mathbf{v}}_{\|}(\mathbf{k})\right|^{2}, \\
E_{\perp}(k) & =\frac{1}{2} \oint \mathrm{d} \Omega_{k} k^{2}\left|\hat{\mathbf{v}}_{\perp}(\mathbf{k})\right|^{2},
\end{aligned}
$$

where $\hat{\mathbf{v}}_{\|}(\mathbf{k})$ and $\hat{\mathbf{v}}_{\perp}(\mathbf{k})$ are the Fourier transforms of the longitudinal and transversal velocity components, respectively. For developed turbulence, it is expected that the the energy spectrum functions follow power laws, $E(k) \propto k^{-\beta}$, in the inertial subrange of wave numbers. A disadvantage of Fourier transforms is that the contributions from small wave numbers are distorted by the non-periodic boundaries of the computational domain. For this reason, we apply Gaussian window functions to the data sets as described in Röpke et al. (2007). Since the data windowing corresponds to a high-pass filter in Fourier space, the range of the energy spectra is constrained to higher wave numbers.

In contrast to the energy spectra, structure functions are two-point velocity correlation functions computed in position space. While the Fourier transforms are performed in Cartesian coordinate systems, we use spherical coordinates for the computation of structure functions, which is convenient to define directions parallel and perpendicular to gravity. Moreover, the computation can be constrained to the interiors of spheres containing a certain amount of burned matter. We define the radial velocity increment by the difference of $\mathbf{v}_{\|}$, i. e., the velocity component in the direction of gravity, at two different positions $\mathbf{r}_{1}$ and $\mathbf{r}_{2}$,

$$
\delta \mathbf{v}_{\text {rad }}=v_{\|}\left(\mathbf{r}_{2}\right)-v_{\|}\left(\mathbf{r}_{1}\right)
$$

The angular velocity increment is defined by the difference of the velocities projected in the directions perpendicular to gravity, i e.,

$$
\delta \mathbf{v}_{\text {ang }}=v_{\perp}\left(\mathbf{r}_{2}\right)-v_{\perp}\left(\mathbf{r}_{1}\right)
$$

Note that these increments do not correspond to longitudinal and transversal velocity increments, because the velocity components are generally not parallel or perpendicular to the spatial separation $\mathbf{r}_{2}-\mathbf{r}_{1}$. However, we think that the above definitions of velocity increments are better suited to the physics of RT-driven turbulence in thermonuclear supernovae. Our proposition is corroborated by the the scaling 
properties of the structure functions that will be presented in the following Section.

The radial and angular structure functions of order $p$ are defined by the averages of the radial and angular velocity increments to the power $p$, respectively:

$$
\begin{aligned}
S_{p, \text { rad }}(\ell) & =\left\langle\left|\delta \mathbf{v}_{\text {rad }}\right|^{p}\right\rangle, \\
S_{p, \text { ang }}(\ell) & =\left\langle\left|\delta \mathbf{v}_{\text {ang }}\right|^{p}\right\rangle,
\end{aligned}
$$

where the length scale $\ell:=\left|\mathbf{r}_{2}-\mathbf{r}_{1}\right|$. There is a large range of length scales which encompasses the turbulent interior of the exploding WD. For fully developed turbulence the structure functions are given by power laws $S_{p \text {,rad }}(\ell) \propto \ell^{\zeta_{p \text {,rad }}}$ and $S_{p, \text { ang }}(\ell) \propto \ell^{\zeta_{p, \text { ang }}}$, where $\zeta_{p \text {,rad }}$ and $\zeta_{p, \text { ang }}$ are characteristic scaling exponents. If we further assume isotropy, $\zeta_{p \text {,rad }} \simeq \zeta_{p \text {.ang }}$, and $\zeta_{p}=p / 3$ according to the theoretical analysis by Kolmogorov (1941). In particular, it follows that the turbulent velocity fluctuation $v^{\prime}(\ell) \propto \ell^{1 / 3}$. For the turbulent flow driven by the RT instability, on the other hand, $v^{\prime}(\ell) \propto \ell^{1 / 2}$ (Davies \& Taylor 1950) corresponding to $\zeta_{p}=p / 2$.

For the numerical computation of the structure functions, one has to take a sufficient large number of sample points that are distributed with uniform probability within a spherical region of prescribed radius in order to achieve converged statistics. This was achieved by a Monte-Carlo-type algorithm, where the total number of sample points was varied and, thereby, convergence was established.

In order to analyze the isotropy of the velocity field close to the flame, we performed calculations in small boxes intersected by the flame. The algorithm is based on the analysis performed by Zingale et al. (2005). To simplify the calculation, the box is placed along a coordinate axis, in our case the $z$-axis, which defines the local direction of gravity. For each cell within the box, the velocity difference $\delta \mathbf{v}$ between the local velocity and the velocity at the center of the box is calculated. Then projected contours of the velocity differences in Fourier space can be constructed, by integrating $\delta \hat{\mathbf{v}}(\mathbf{k})$ over circles of radius $k_{\rho}=\sqrt{k_{x}^{2}+k_{y}^{2}}$ in planes perpendicular to the $z$-component of $\mathbf{k}$. This procedure was applied for several positions of the box center corresponding to different fractions of burned matter in the box.

\section{RESULTS}

\subsection{Energy spectra}

We plot the energy spectra as functions of the normalized wave number $k_{\mathrm{n}}=512 \Delta_{0}(t) k / \pi$ for $16 \leq k_{\mathrm{n}} \leq 512$, where $\Delta_{0}(t)$ is the size of the cells in the uniform part of the grid at time $t$. We have $\Delta_{0}(t)=2.93$ and $14.69 \mathrm{~km}$ at times $t=0.5$ and 1.0 seconds, respectively. Note that wave numbers $k_{\mathrm{n}}<32$ are obscured by data windowing (see Section 2). The computed energy spectrum functions at 0.5 seconds are shown as black curves in Fig. 1 (a). The thin gray lines corresponds to power-law fits and the dashed line indicates the expected spectrum according to the Kolmogorov theory with an exponent $\beta=5 / 3$. For the longitudinal spectrum function, we find $\beta \approx 1.64$ for high wave numbers, which is in good agreement with the Kolmogorov theory. The longitudinal spectrum possibly stiffens toward lower wave numbers, but the accuracy of the computed spectra does not allow for a conclusive result. We exclude the lower part of wavenumbers from the fit, because the slope becomes steeper than a Kolmogorov spectrum for $k_{\mathrm{n}} \lesssim 64$. The transversal spectrum is slightly steeper $(\beta \approx 1.71)$, but close to a Kolmogorov spectrum for the whole range of wave numbers. These results were corroborated by the computation of second-order structure functions with much higher accuracy (see section 3.2). We also note that the Kolmogorov scaling for higher wave numbers agrees with the findings of Röpke et al. (2007), where turbulence energy spectra were computed without splitting the velocity field. This is in accordance with the expectation that the radial expansion will mostly affect low wave numbers modes. At $t=1.0$ seconds, on the other hand, the longitudinal spectrum has an exponent $\beta \approx 1.97$ over the entire range of wave numbers. In contrast, the transverse spectrum is much shallower. The exponent $\beta=2$ corresponds to RT scaling, because $E(k) \propto k^{-2}$ implies $\delta v(\ell) \propto \ell^{1 / 2}$. Consequently, it appears that the velocity component in the direction of gravity is dominated by the RT instability even at the smallest numerically resolved scales in the late phase of the explosion, while Kolmogorov scaling is found for the velocity component perpendicular to gravity.

\subsection{Velocity structure functions}

For the computation of the structure functions following equation (4a) and (4b), we chose a spherical region in which $90 \%$ of the material was burned. This choice of the region resulted from the requirement of encompassing the bulk of turbulence at a given time, while excluding the outer, non-turbulent regions of the white dwarf. Fig. 22 shows double-logarithmic plots of the radial (solid curve) and angular (dashed curve) structure functions of second order at different times. In all cases up to $t=0.7$ seconds, a scaling exponent $\zeta_{2}$ close to $2 / 3$ is found for the range of length scales $\ell \lesssim 10 \mathrm{~km}$. Moreover, $S_{p \text {,rad }}(\ell) \approx S_{p \text {,ang }}(\ell)$, which indicates isotropy at small length scales. For larger length scales, on the other hand, the radial structure functions $S_{p, \text { rad }}(\ell)$ obey a scaling law with an exponent $\zeta_{2 \text {,rad }} \approx 1$, whereas $S_{p \text {,ang }}(\ell)$ still follows Kolmogorov scaling. From $\zeta_{2, \text { rad }} \approx 1$, it follows that $v^{\prime}(\ell) \propto \ell^{1 / 2}$. As outlined in section 2 this corresponds to RT scaling. For this reason, the change of slope of the radial structure function indicates a transition from the inertial-range turbulence cascade to the regime of RT instabilities at a length scale $\ell_{\mathrm{K} / \mathrm{RT}}$ approaching $\approx 14 \mathrm{~km}$ in the course of the explosion. Thus, our analysis confirms the estimate by Niemeyer \& Woosley (1997). As a result of the overall expansion of the co-moving grid, the transition length drops below the numerical resolution of the simulation after $\sim 0.7$ to 1.0 seconds. The time evolution of $\ell_{\mathrm{K} / \mathrm{RT}}$ is further illustrated in Fig. 3 . where the second order structure functions are plotted for all data sets, for which $\ell_{\mathrm{K} / \mathrm{RT}}$ is numerically resolved. While in Fig. 2 the range of length scales is adjusted to the size of the co-moving grid, a fixed range of length scales is used in Fig. 3 Additionally, the typical mass density in the vicinity of the flame front is specified for each instant of time. In agreement with Fig. 1 of Niemeyer \& Woosley (1997), Fig. 2 and 3 show that $\ell_{\mathrm{K} / \mathrm{RT}}$ becomes smaller with decreasing density (and advancing time). Remarkably, it appears that $\ell_{\mathrm{K} / \mathrm{RT}}$ approaches an asymptotic value, but we cannot investigate this behavior for $t>0.8$ seconds. To obtain more properties of $\ell_{\mathrm{K} / \mathrm{RT}}$ additional high-resolved numerical simulations are needed, in which $\ell_{\mathrm{K} / \mathrm{RT}}$ can be tracked for a longer time.

In Figure 4 the structure functions up to the sixth order are plotted for $t=0.5,0.6$ and 0.7 seconds. As one can see from the scaling exponents listed in Figure 3, $\zeta_{3}$ is close to unity for the angular structure functions and for the radial structure 


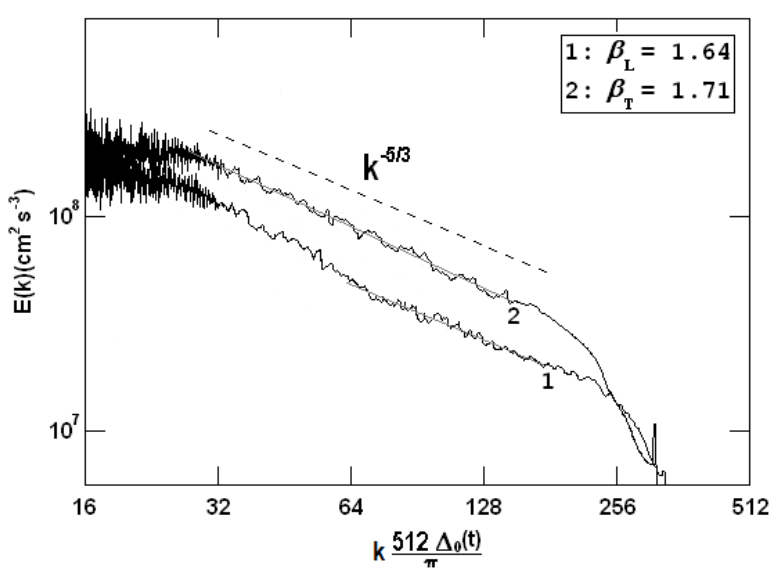

(a) $t=0.5$ seconds

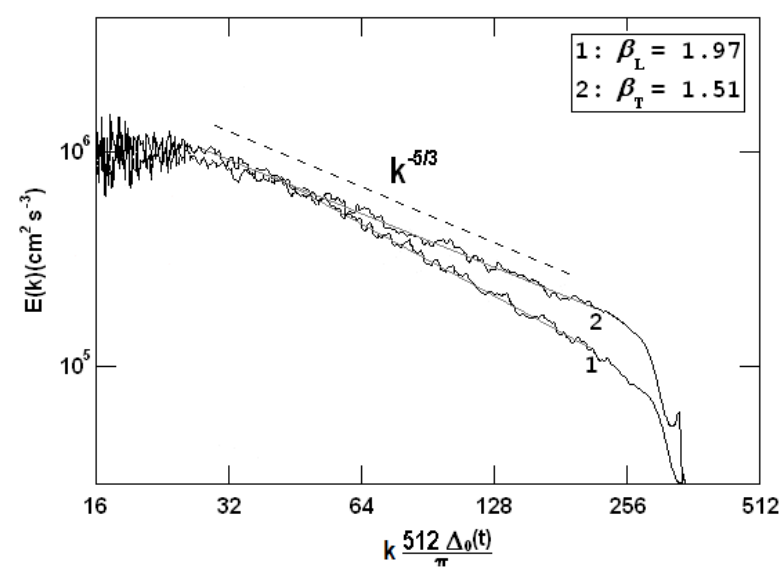

(b) $\mathrm{t}=1.0$ seconds

FIG. 1. - Longitudinal (1) and transversal (2) energy spectrum function (black curve) at $t=0.5$ (a) and $t=1.0$ (b) seconds in comparison with the Kolmogorov energy spectrum (dashed line).

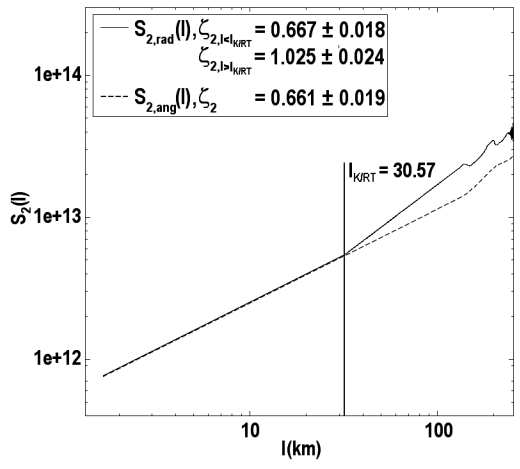

(a) $\mathrm{t}=0.3$ seconds

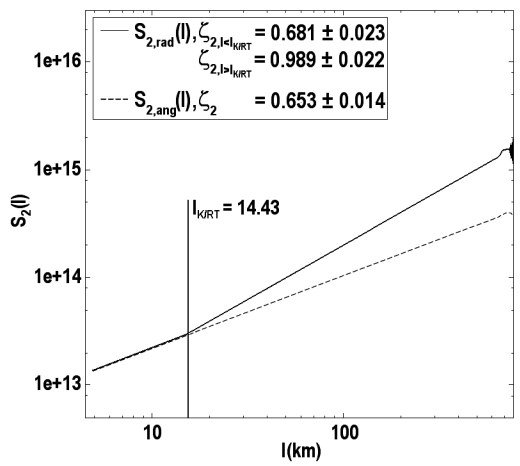

(d) $\mathrm{t}=0.6$ seconds

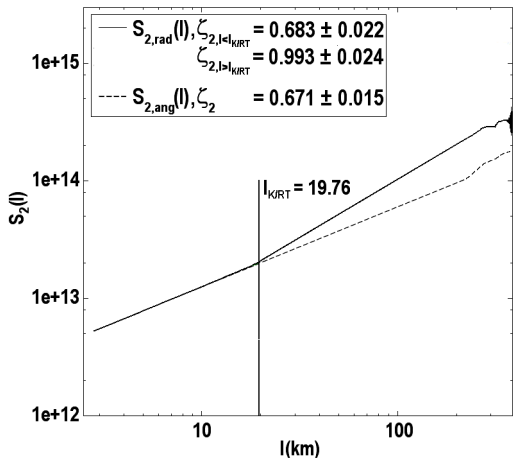

(b) $\mathrm{t}=0.4$ seconds

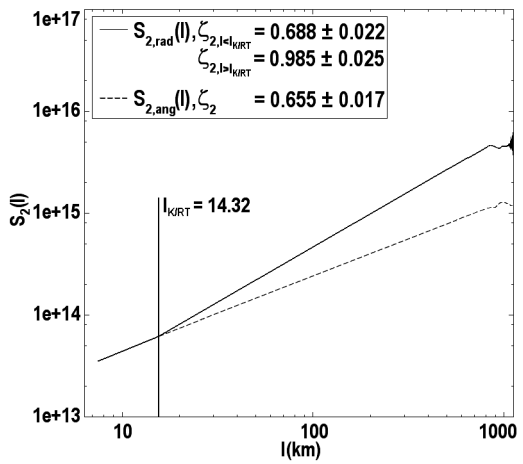

(e) $\mathrm{t}=0.7$ seconds

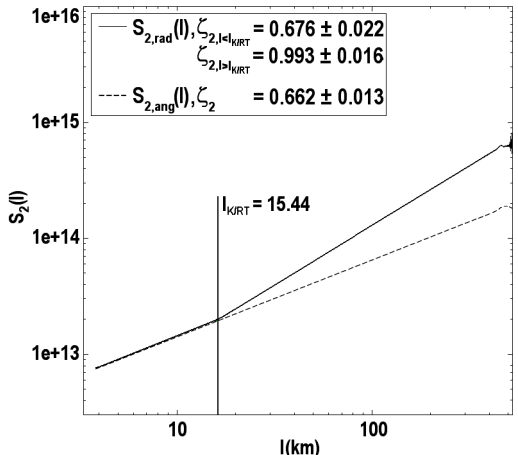

(c) $t=0.5$ seconds

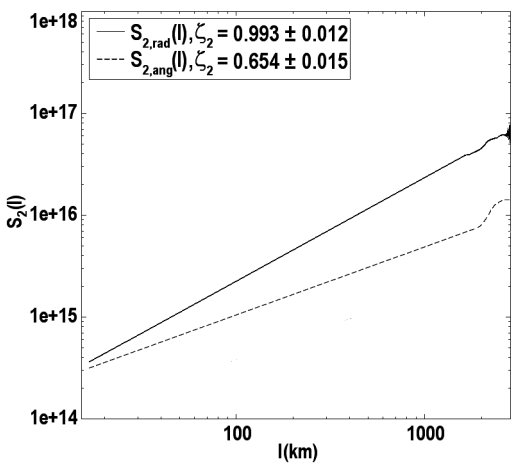

(f) $\mathrm{t}=1.0$ seconds

FIG. 2.- Radial (solid curve) and angular (dashed curve) 2nd-order structure function with the determined transition length scale.

functions in the subrange $\ell<\ell_{\mathrm{K} / \mathrm{RT}}$. This result is consistent with the Kolmogorov theory (see Frisch 1995). For $\ell>\ell_{\mathrm{K} / \mathrm{RT}}$, on the other hand, $\zeta_{3, \text { rad }} \approx 1.5$. Remarkably, it appears that for all $p \leq 6$ the slopes of the radial structure functions are steeper by a factor of 1.5 at length scales greater than $\ell_{\mathrm{K} / \mathrm{RT}}$. This suggests that the ratio $Z_{p}:=\zeta_{p} / \zeta_{3}$ is approximately equal for $S_{p, \text { rad }}\left(\ell<\ell_{\mathrm{K} / \mathrm{RT}}\right)$ and $S_{p, \text { rad }}\left(\ell>\ell_{\mathrm{K} / \mathrm{RT}}\right)$. Indeed, the relative scaling exponents of the radial structure functions in both subranges are quite close (see Table 11). There are theoretical arguments (Dubrulle 1994) as well as numerical investigations (Benzi et al. 1993) in support of the fundamen- tal significance of relative scaling exponents. But it has not been noticed before that RT-driven velocity fluctuations exhibit statistical properties that are equivalent to the properties of isotropic, inertial-range turbulence in terms of relative scalings. It is known that the calculation of higher-order exponents becomes increasingly uncertain due to sampling errors (Frisch 1995). For this reason, we do not consider the relatively high discrepancies between the results for $p \geq 5$ to be significant. Comparing the relative scaling exponents of the radial and the angular structure functions in the subrange $\ell>\ell_{\mathrm{K} / \mathrm{RT}}$, on the other hand, we find very good agreement. 
TABLE 1

RELATIVE SCALING EXPONENTS $Z_{p}$ AT THREE DIFFERENT INSTANTS OF TIME

\begin{tabular}{cccccccc}
\hline \hline & $t[s]$ & $p=1$ & $p=2$ & $p=3$ & $p=4$ & $p=5$ & $p=6$ \\
\hline \multirow{2}{*}{$Z_{p, \text { rad }}$ for $\ell<\ell_{\mathrm{K} / \mathrm{RT}}$} & 0.5 & $0.344 \pm 0.016$ & $0.676 \pm 0.027$ & $1.0 \pm 0.037$ & $1.309 \pm 0.050$ & $1.597 \pm 0.058$ & $1.860 \pm 0.068$ \\
& 0.6 & $0.341 \pm 0.016$ & $0.676 \pm 0.030$ & $1.0 \pm 0.046$ & $1.304 \pm 0.057$ & $1.593 \pm 0.071$ & $1.850 \pm 0.082$ \\
& 0.7 & $0.347 \pm 0.019$ & $0.676 \pm 0.029$ & $1.0 \pm 0.038$ & $1.302 \pm 0.052$ & $1.584 \pm 0.060$ & $1.842 \pm 0.074$ \\
\hline \multirow{2}{*}{$Z_{p, \text { rad }}$ for $\ell>\ell_{\mathrm{K} / \mathrm{RT}}$} & 0.5 & $0.339 \pm 0.009$ & $0.674 \pm 0.015$ & $1.0 \pm 0.021$ & $1.315 \pm 0.027$ & $1.615 \pm 0.033$ & $1.900 \pm 0.038$ \\
& 0.6 & $0.338 \pm 0.011$ & $0.674 \pm 0.019$ & $1.0 \pm 0.027$ & $1.316 \pm 0.033$ & $1.613 \pm 0.042$ & $1.896 \pm 0.050$ \\
& 0.7 & $0.338 \pm 0.011$ & $0.674 \pm 0.019$ & $1.0 \pm 0.027$ & $1.315 \pm 0.035$ & $1.615 \pm 0.043$ & $1.898 \pm 0.050$ \\
\hline \multirow{2}{*}{$Z_{p, \text { ang }}$} & 0.5 & $0.336 \pm 0.008$ & $0.670 \pm 0.016$ & $1.0 \pm 0.024$ & $1.323 \pm 0.034$ & $1.636 \pm 0.042$ & $1.920 \pm 0.050$ \\
& 0.6 & $0.335 \pm 0.013$ & $0.668 \pm 0.018$ & $1.0 \pm 0.026$ & $1.319 \pm 0.034$ & $1.625 \pm 0.045$ & $1.919 \pm 0.050$ \\
& 0.7 & $0.335 \pm 0.009$ & $0.670 \pm 0.016$ & $1.0 \pm 0.022$ & $1.323 \pm 0.030$ & $1.635 \pm 0.035$ & $1.934 \pm 0.041$ \\
\hline
\end{tabular}

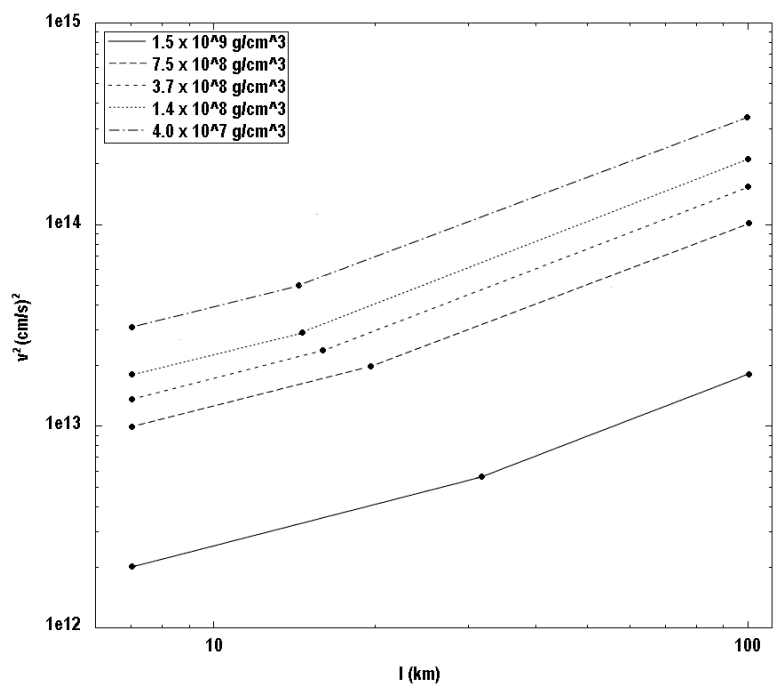

FIG. 3.-2nd order radial structure functions with the transition length (big dots) and corresponding density on the flame front.

We will concentrate on these scaling exponents in the following.

\subsection{Local isotropy of the velocity fluctuations}

The contour lines of the Fourier-transformed velocity field at 0.5 seconds inside a box as described in Section 2 are plotted in Fig 5. The box is positioned such that $50 \%$ of the enclosed matter is burned. The thick dashed line indicate the wave number $2 \pi / \ell_{\mathrm{K} / \mathrm{RT}}$ corresponding to the transition length obtained from the calculation of the radial structure functions. For smaller wave numbers (i. e., $\ell \gtrsim \ell_{\mathrm{RT}}$ ), the contours of $\delta \hat{\mathbf{v}}(\mathbf{k})$ are clearly anisotropic. One can see that a given velocity difference in the $z$-direction spans a smaller range of wave numbers than in the directions perpendicular to the $z$-axis. This corresponds to a steeper slope of the velocity fluctuations in the radial direction, which is approximately given by the $z$-direction. Toward higher wave numbers $\left(\ell \lesssim \ell_{\mathrm{RT}}\right)$, the anisotropy of the contours decreases, but we do not find perfect isotropy. This might indicate residual small-scale anisotropy in the vicinity of the flame front, which can be caused by the intermittency of turbulence. However, it could also be a spurious effect due to the misalignment between the $z$-axis and the radial direction at off-center positions within the box.

\section{CONCLUSION}

We investigated the scaling properties of turbulence in a high-resolution simulation of a Type Ia supernova based on the pure deflagration model (Röpke et al.|2007). Both energy spectrum functions and structure functions of second order were computed. The results of this study are as follows:

1. The velocity fluctuations in the radial direction, i. e., the direction of gravity, follow Kolmogorov scaling at length scales smaller than a certain transition length $\ell_{\mathrm{K} / \mathrm{RT}} \sim 10 \mathrm{~km}$. Only at length scales greater than $\ell_{\mathrm{K} / \mathrm{RT}}$, the radial velocity fluctuations are dominated by the scaling law of the Rayleigh-Taylor instability. This behaviour was predicted by Niemeyer \& Woosley (1997).

2. The velocity fluctuations in angular directions, i. e., perpendicular to gravity, obey Kolmogorov scaling over the entire range of numerically resolved length scales.

3. For $\ell \lesssim \ell_{\mathrm{K} / \mathrm{RT}}$, the magnitudes of the radial and angular velocity fluctuations are nearly equal. For this reason, small-scale turbulence appears to be statistically isotropic.

4. Fourier analysis of the velocity fluctuations in a small region near the flame surface allows for slight residual anisotropies at the smallest resolved scales.

As regards the interpretation of our results, a possible cause for concern is that Kolmogorov scaling in the radial direction is only found for a relatively narrow range of length scales greater than the numerical cutoff length. It is known that these scales are affected by numerical dissipation and, particularly, by the bottleneck effect (Schmidt et al. 2006). Thus, the flattening of the turbulence energy spectrum might be artificial. However, in this case, no significant flattening should be observed for the corresponding structure functions which are much less affected by the bottleneck effect. The scaling laws implied by the radial energy spectra and structure functions are fully consistent at small length scales and the transversal spectra show no flattening at all. In consequence, we are confident that the scaling laws are genuine. A possible explanation for the absence of the bottleneck effect is that turbulence does not reach a statistically stationary state in a supernova explosion.

Yet another issue is that Kolmogorov scaling might be enforced by the subgrid scale model that was used in the simulation. However, other than the RT-based models used by Gamezo et al. (2003), this SGS model does neither presume any given scaling of turbulence nor does it imprint such a scaling on the numerically resolved flow. A potential problem for the SGS model is the possible lack of isotropy near the flame surface. However, there is certainly no pronounced anisotropy at the smallest resolved scales if $\Delta_{0}(t)<\ell_{\mathrm{K} / \mathrm{RT}}$, and statistical isotropy is found for the bulk of turbulent regions. Only in the 


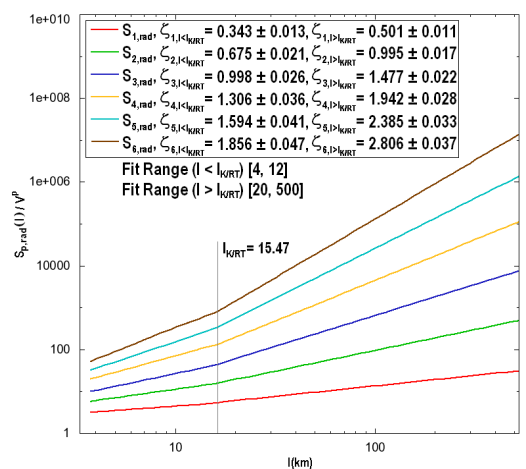

(a) $\mathrm{t}=0.5$ seconds, radial

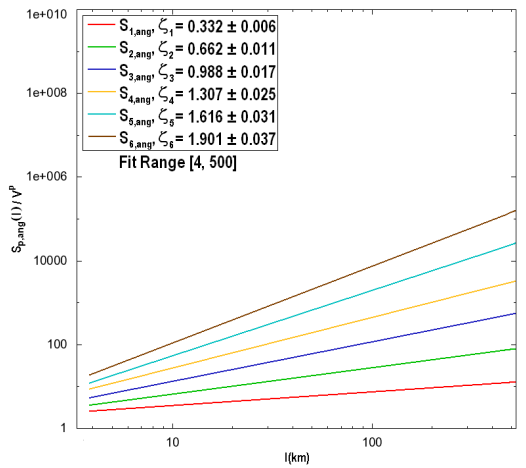

(d) $\mathrm{t}=0.5$ seconds, angular

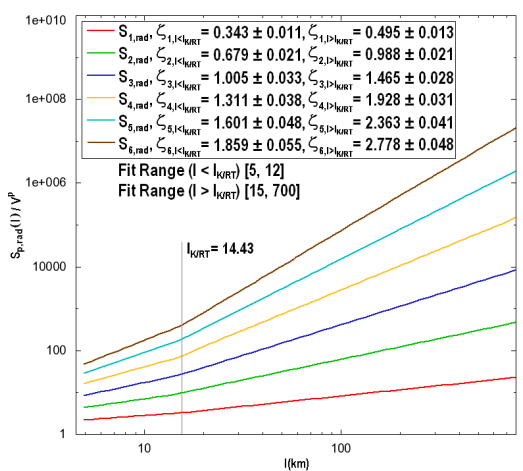

(b) $\mathrm{t}=0.6$ seconds, radial

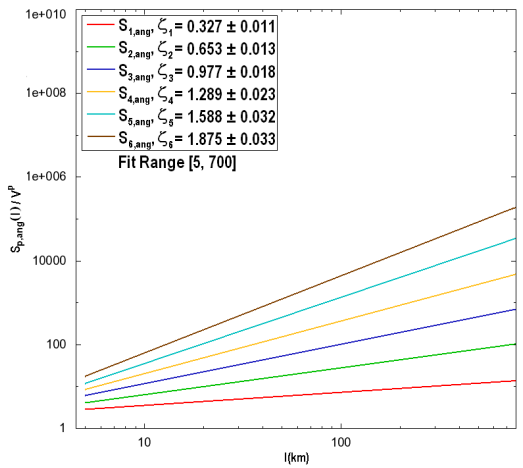

(e) $\mathrm{t}=0.6$ seconds, angular

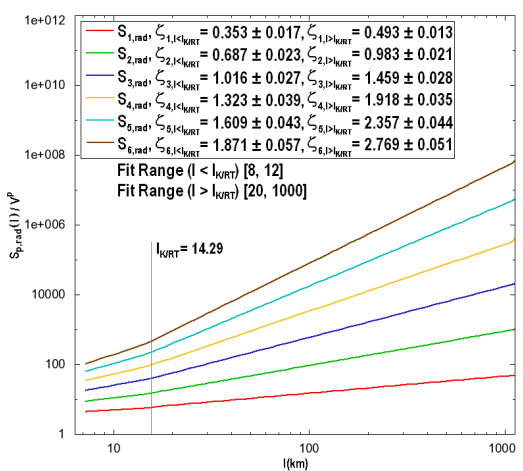

(c) $\mathrm{t}=0.7$ seconds, radial

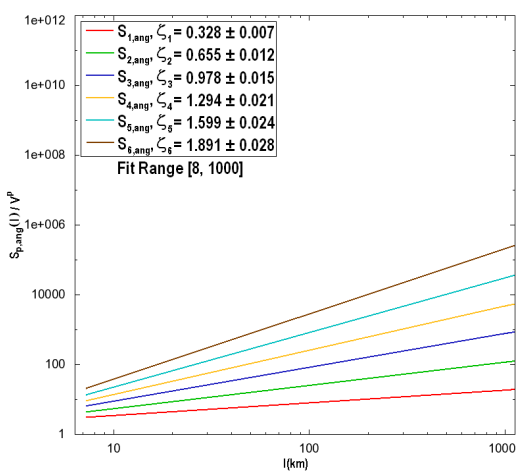

(f) $\mathrm{t}=0.7$ seconds, angular

FIG. 4.- Radial and angular structure function up to the sixth order with the corresponding scaling exponents. For the radial structure functions, the transition length from Kolmogorov to Rayleigh-Taylor scaling $\ell_{\mathrm{K} / \mathrm{RT}}$ is indicated.

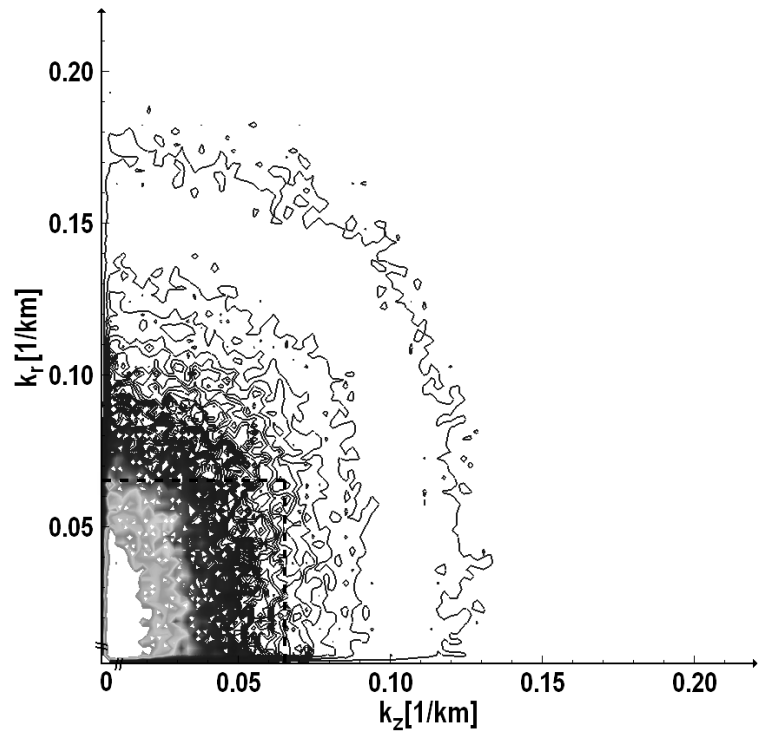

FIG. 5.- Contour plots of the Fourier-transformed velocity differences inside a small box intersected by the flame front at 0.5 seconds.

late phase of the explosion, when the transition length $\ell_{\mathrm{K} / \mathrm{RT}}$ becomes smaller than the numerical resolution and the resolved small-scale turbulence definitely becomes anisotropic, the notion of SGS turbulence energy cannot be strictly justified. One should note, however, that this point more or less coincides with the time when a deflagration-to-detonation transition is expected to occur (Gamezo et al. 2005; Röpke \& Niemeyer 2007). Apart from that, the production of turbulence energy by unresolved buoyancy effects is heuristically included in the SGS model. In conclusion, the SGS turbulence energy model by Schmidt et al. (2006) applies to the major part of the explosive burning in the deflagration phase of a Type Ia supernova explosion, but there is no regime for which a pure RT-scaling model holds.

After settling the issue of turbulence scaling in the deflagration phase of a Type Ia supernova in the present article, we mention that the occurrence of deflagration-to-detonation transitions can be constrained on the basis of the deflagration model. For the DDT mechanism to operate, strong turbulence is necessary in late phases of the burning (e.g. Woosley 2007). Röpke (2007) found that this may indeed be realized in deflagration models of SNe Ia with low (but not vanishing) probability. In order to better quantify this intermittency effect, higher-order structure functions have to be computed. Fitting intermittency models to the numerically determined scaling exponents (as proposed by Pan et al. 2008), the probability of strong turbulent velocity fluctuations at any instant of time can be estimated. This analysis will be presented in a future publication.

The research of F.K.R. is supported through the Emmy Noether Program of the German Research Foundation (DFG; RO 3676/1-1).

\section{REFERENCES}

Benzi, R., Ciliberto, S., Tripiccione, R., Baudet, C., Massaioli, F., \& Succi, S. 1993, Phys. Rev. E, 48, 29
Davies, R. M., \& Taylor, G. 1950, Proc. Roy. Soc. London A, 200, 375 
Dubrulle, B. 1994, Phys. Rev. Lett., 73, 959

Frisch, U. 1995, Turbulence. The legacy of A.N. Kolmogorov (Cambridge: Cambridge University Press, lc1995)

Gamezo, V. N., Khokhlov, A. M., \& Oran, E. S. 2005, ApJ, 623, 337

Gamezo, V. N., Khokhlov, A. M., Oran, E. S., Chtchelkanova, A. Y., \& Rosenberg, R. O. 2003, Science, 299, 77

Hillebrandt, W., \& Niemeyer, J. C. 2000, ARA\&A, 38, 19

Kolmogorov, A. 1941, Akademiia Nauk SSSR Doklady, 30, 301

Niemeyer, J. C., \& Hillebrandt, W. 1995, ApJ, 452, 769

Niemeyer, J. C., \& Kerstein, A. R. 1997, New Astronomy, 2, 239

Niemeyer, J. C., \& Woosley, S. E. 1997, ApJ, 475, 740

Pan, L., Wheeler, J. C., \& Scalo, J. 2008, ApJ, 681, 470

Reinecke, M., Hillebrandt, W., \& Niemeyer, J. C. 2002, A\&A, 391, 1167

Röpke, F. K. 2005, A\&A, 432, 969

Röpke, F. K. 2007, ApJ, 668, 1103
Röpke, F. K., Hillebrandt, W., Schmidt, W., Niemeyer, J. C., Blinnikov, S. I., \& Mazzali, P. A. 2007, ApJ, 668, 1132

Röpke, F. K., \& Niemeyer, J. C. 2007, A\&A, 464, 683

Röpke, F. K., \& Schmidt, W. 2008, Lecture Notes in Physics, Vol. 756, Turbulent combustion in thermonuclear supernovae (Springer), 255-289

Schmidt, W., Hillebrandt, W., \& Niemeyer, J. C. 2006, Comp. Fluids., 35, 353

Schmidt, W., Niemeyer, J. C., \& Hillebrandt, W. 2006, A\&A, 450, 265

Sharp, D. H. 1984, Physica D Nonlinear Phenomena, 12, 3

Woosley, S. E. 2007, ApJ, 668, 1109

Zingale, M., Woosley, S. E., Rendleman, C. A., Day, M. S., \& Bell, J. B. 2005, ApJ, 632, 1021 